\title{
Time-Domain-Multiplexed Measurement-Based Quantum Operations with 25-MHz Clock Frequency
}

\author{
Warit Asavanant $\odot,{ }^{1, *}$ Baramee Charoensombutamon $\odot,{ }^{1}$ Shota Yokoyama $\odot,{ }^{2}$ Takeru Ebihara, ${ }^{1}$ \\ Tomohiro Nakamura, ${ }^{1}$ Rafael N. Alexander $\odot,{ }^{3,4}$ Mamoru Endo $\odot,{ }^{1}$ Jun-ichi Yoshikawa, ${ }^{1}$ \\ Nicolas C. Menicucci® ${ }^{4}$ Hidehiro Yonezawa $\odot,{ }^{2}$ and Akira Furusawa $\odot^{1,5, \dagger}$ \\ ${ }^{1}$ Department of Applied Physics, School of Engineering, The University of Tokyo, 7-3-1 Hongo, Bunkyo-ku, \\ Tokyo 113-8656, Japan \\ ${ }^{2}$ Centre for Quantum Computation and Communication Technology, School of Engineering and Information \\ Technology, University of New South Wales, Canberra, Australian Capital Territory 2600, Australia \\ ${ }^{3}$ Center for Quantum Information and Control, Department of Physics and Astronomy, University of New Mexico, \\ Albuquerque, New Mexico 87131, USA \\ ${ }^{4}$ Centre for Quantum Computation and Communication Technology, School of Science, RMIT University, \\ Melbourne, Victoria 3001, Australia \\ ${ }^{5}$ Optical Quantum Computing Research Team, RIKEN Center for Quantum Computing, 2-1 Hirosawa, Wako, \\ Saitama 351-0198, Japan
}

(Received 14 April 2021; revised 25 May 2021; accepted 11 August 2021; published 2 September 2021)

Continuous-variable optical quantum computation has seen much progress in recent years. In particular, cluster states - the universal resource for measurement-based quantum computation - have been realized in a scalable fashion using the time-domain multiplexing method. To utilize the cluster states in actual quantum computation, the measurement bases need to be programmed according to the desired computation. In addition, as the information is encoded in time in the time-domain multiplexing method, the measurement bases must be dynamically changed in time to fully utilize the large-scale cluster states. Here we report demonstrations of quantum operations using time-domain-multiplexed cluster states with a clock frequency of $25 \mathrm{MHz}$. This is achieved by our combining the clusterstate-generation setup with the setup to change the measurement basis in the time domain. We also formulate a method to evaluate and verify continuous-variable operations where the quantum entanglements in the cluster states are utilized. Therefore, we demonstrate the implementation of quantum operations on scalable continuous-variable cluster-state architectures. The results in this work are compatible with the developing nonlinear feedforward and non-Gaussian state generation technology, which brings the realization of the large-scale fault-tolerant universal optical quantum computer closer to reality.

DOI: 10.1103/PhysRevApplied.16.034005

\section{INTRODUCTION}

Quantum computation has many promising applications [1]. To fully harness the power of the quantum computer, however, it is important to realize a large-scale quantum computer with universality and fault tolerance. In this regard, an approach to quantum computation called "measurement-based quantum computation" (MBQC) is

*warit@alice.t.u-tokyo.ac.jp

†akiraf@ap.t.u-tokyo.ac.jp

Published by the American Physical Society under the terms of the Creative Commons Attribution 4.0 International license. Further distribution of this work must maintain attribution to the author(s) and the published article's title, journal citation, and DOI. one of the most-promising candidates [2,3]. Figure 1(a) shows a schematic diagram of MBQC. In MBQC, a multiqubit quantum entanglement called a "cluster state" is first prepared. After the cluster states are prepared, the quantum operations are implemented via single-qubit measurements and feedforward operations. MBQC replaces the need to prepare and interface a large number of qubits with cluster-state preparation, single-qubit measurements, and feedforward operations. Many experimental studies toward realization of MBQC have been performed in various physical systems [4-12]. Among these systems, continuous-variable (CV) MBQC using optical modes multiplexed in the time domain [13] is currently the leading candidate in terms of scalability; by multiplexing multiple optical modes on the same beam, large-scale cluster states - 10000-mode [4] and 1000000 -mode [5] 
(a) ।

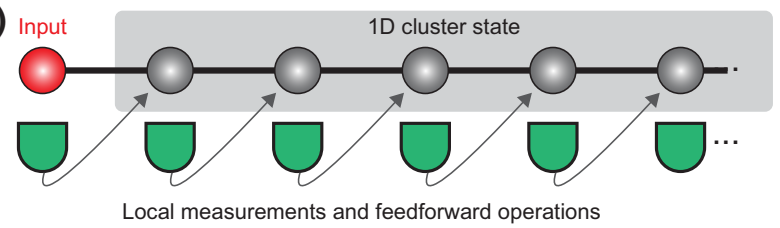

(b) Input

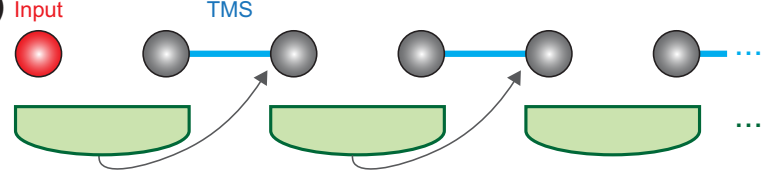

Nonlocal measurements and feedforward operations

FIG. 1. Conceptual diagram of measurement-based quantum computation. (a) The original approach using half-teleportation. (b) The equivalent approach based on sequential teleportation that is used in the time-domain multiplexing method. $1 \mathrm{D}$, one dimensional; TMS, two-mode-squeezed state.

one-dimensional cluster states and universal resources for multi-input MBQC called "two-dimensional cluster states" $[6,7]$ - have already been demonstrated.

With the realization of cluster states, we have to make measurements on the cluster states to realize quantum operations. Although the encoding of the optical mode in a temporally localized wave packet allows large-scale generation of cluster states via repeated use of the same optical components, this complicates the measurements on cluster states. The measurement bases must now be changed dynamically in time, in contrast to the usual optical cluster states, where each mode is spatially separated and the measurement bases can be fixed [12]. In general, any type of multiplexing involves the sharing of a degree of freedom to reduce the number of optical components required. As an example additional to time-domain multiplexing, all the modes of the cluster states in the frequency domain multiplexed are generated at the same timing on the same beam [10]. Even supposing a large-scale cluster state can be generated, accessing each mode and efficiently implementing quantum operations is not trivial. In addition, unlike the qubit system, where standard procedures for evaluation of the quantum operations such as randomized benchmarking [14] have been established even for MBQC [15], the experimental method to benchmark CV systems has not been fully developed yet.

In this work, we present a demonstration of one-mode Gaussian operations multiplexed in the time domain by changing the measurement basis dynamically. This demonstration establishes the experimental method to use largescale time-domain-multiplexed cluster states in actual quantum operations, a fundamental component for realizing CV quantum computation. Experimentally, as the size of our wave packet is $40 \mathrm{~ns}$, we can implement quantum operations with a clock frequency of $25 \mathrm{MHz}$ using the current system. To evaluate the implemented operations, we utilize the quantum entanglements in the system and verify that the quantum operations are implemented according to the programmed measurement bases. Using this quantum entanglement, we also verify that the preshared entanglement persists between different modes even after quantum operations are implemented. To show the capability of the multistep operations, we implement sequential teleportation and observe that its behavior matches the theoretical predictions up to 100 steps. This method for the evaluation of the multistep operation is in the same spirit as randomized benchmarking, where the behavior of the noise when multistep operations are implemented is used to quantify the quality of the quantum operations. Our work is an important milestone in $\mathrm{CV}$ quantum computation. Largescale fault-tolerant universal quantum computation can be achieved by combining nonlinear feedforward operations [16] and non-Gaussian ancillary states [17,18] with the method in this work.

This paper is structured as follows. Section II reviews the theory regarding CV quantum operations. Section III discusses the experimental setup. The evaluation method using quantum entanglement that is developed in this work is explained in Sec. IV. The experimental results are presented in Sec. V. Future prospects and conclusions are given in Sec. VI.

\section{CV QUANTUM OPERATIONS}

In this section, we briefly explain the CV quantum operations in our system.

In a CV system, the physical quantities of interest are quadratures: $\hat{x}$ and $\hat{p}$. For each temporal wave packet, the quadrature operators satisfy $\left[\hat{x}_{k}, \hat{p}_{l}\right]=i \hbar \delta_{k l}$ where $k$ and $l$ are the indices of the wave packet. Linear combinations of quadrature operators $\hat{x}$ and $\hat{p}$ are measured by homodyne measurements. We define $\hat{x}(\theta) \equiv(\cos \theta) \hat{x}+(\sin \theta) \hat{p}$ and $\hat{p}(\theta) \equiv(\cos \theta) \hat{p}-(\sin \theta) \hat{x}$.

Quantum operations in a CV system are categorized into two types: Gaussian operations and non-Gaussian operations. Gaussian operations are the operations that are linear transformations of the quadrature operators in the Heisenberg picture. Arbitrary multimode Gaussian operations can be implemented via homodyne measurements on the $\mathrm{CV}$ cluster states with an appropriate graph structure $[3,6,7]$. If we restrict ourselves to one-mode Gaussian operations, arbitrary one-mode Gaussian operations have the form

$$
\left(\begin{array}{l}
\hat{x}_{\text {out }} \\
\hat{p}_{\text {out }}
\end{array}\right)=\left(\begin{array}{ll}
S_{11} & S_{12} \\
S_{21} & S_{22}
\end{array}\right)\left(\begin{array}{l}
\hat{x}_{\text {in }} \\
\hat{p}_{\text {in }}
\end{array}\right)+\left(\begin{array}{c}
x_{0} \\
p_{0}
\end{array}\right),
$$

where $S_{i j}$ are elements of a symplectic matrix and $x_{0}$ and $p_{0}$ are real numbers corresponding to the displacement on the phase space. The commonly used one-mode Gaussian operations (besides displacements) are phase rotation $\mathbf{R}(\phi)$, squeezing $\mathbf{S}(\phi)$, and shear $\mathbf{P}(\phi)$, whose symplectic 
matrices are parametrized as follows:

$$
\begin{aligned}
& \mathbf{R}(\phi)=\left(\begin{array}{cc}
\cos \phi & \sin \phi \\
-\sin \phi & \cos \phi
\end{array}\right), \\
& \mathbf{S}(\phi)=\left(\begin{array}{cc}
1 / \tan \phi & 0 \\
0 & \tan \phi
\end{array}\right), \\
& \mathbf{P}(\phi)=\left(\begin{array}{cc}
1 & 0 \\
2 \tan \phi & 1
\end{array}\right) .
\end{aligned}
$$

In the original formulation of CV MBQC [3], the quantum operations using the $\mathrm{CV}$ system are formulated on the basis of the CV half-teleportation circuit. For the time-domain-multiplexed CV cluster states, however, it was shown that the quantum operations can be equivalently considered as the sequential $\mathrm{CV}$ full teleportation using the two-mode entangled states called "twomode-squeezed states" (also called "Einstein-PodolskyRosen states") [4]. Figure 1(b) shows a schematic diagram of this concept. When the measurement basis of the two homodyne measurements in the quantum teleportation circuit is selected as $\hat{x}^{A}\left(\theta^{A}\right)=\left(\cos \theta^{A}\right) \hat{x}^{A}+$ $\left(\sin \theta^{A}\right) \hat{p}^{A}$ and $\hat{x}^{B}\left(\theta^{B}\right)=\left(\cos \theta^{B}\right) \hat{x}^{B}+\left(\sin \theta^{B}\right) \hat{p}^{B}$, the CV full-teleportation circuit implements the following operation in the infinite squeezing limits:

$$
\left(\begin{array}{l}
\hat{x}_{\text {out }} \\
\hat{p}_{\text {out }}
\end{array}\right)=\mathbf{V}\left(\theta^{A}, \theta^{B}\right)\left(\begin{array}{l}
\hat{x}_{\text {in }} \\
\hat{p}_{\text {in }}
\end{array}\right),
$$

where

$$
\begin{aligned}
\mathbf{V}\left(\theta^{A}, \theta^{B}\right) & =\mathbf{R}\left(\theta_{+}-\frac{\pi}{2}\right) \mathbf{S}\left(\theta_{-}\right) \mathbf{R}\left(\theta_{+}\right), \\
\theta_{ \pm} & =\frac{\theta^{B} \pm \theta^{A}}{2}
\end{aligned}
$$

By selection of an appropriate combination of $\theta^{A}$ and $\theta^{B}$, various Gaussian operations can be implemented. The relations between the measurement bases and the implemented Gaussian operations that are demonstrated in this work are as follows:

$$
\begin{aligned}
\mathbf{R}(\phi) & =\mathbf{V}\left(\frac{\phi}{2}, \frac{\phi}{2}+\frac{\pi}{2}\right), \\
\mathbf{R}\left(\frac{\pi}{2}\right) \mathbf{S}(\phi) & =\mathbf{V}(\phi,-\phi), \\
\mathbf{P}(\phi) & =\mathbf{V}\left(0, \frac{\pi}{2}-\phi\right) .
\end{aligned}
$$

When the squeezing resource is finite and the optical losses are absent, additional noise terms appear in Eq. (5) as

$$
\left(\begin{array}{l}
\hat{x}_{\text {out }} \\
\hat{p}_{\text {out }}
\end{array}\right)=\mathbf{V}\left(\theta^{A}, \theta^{B}\right)\left(\begin{array}{l}
\hat{x}_{\text {in }} \\
\hat{p}_{\text {in }}
\end{array}\right)+\sqrt{2}\left(\begin{array}{l}
\hat{x}_{\text {anc }, 1} e^{-r_{x}} \\
\hat{p}_{\text {anc }, 2} e^{-r_{p}}
\end{array}\right),
$$

where the label "anc" refers to the ancillary squeezed states, and $r_{x}$ and $r_{p}$ are the squeezing parameters of the initial ancillary squeezed states used to generate the two-mode-squeezed state.

To implement universal MBQC, non-Gaussian operations are also necessary [19], in addition to the multimode Gaussian operations. If we can implement arbitrary Gaussian operations, then we need only to add appropriate non-Gaussian ancillary states to implement non-Gaussian operations [20-22]. Therefore, the only measurements that must be implemented on the CV cluster state to implement quantum operations are homodyne measurements.

\section{EXPERIMENT SETUP}

Figure 2 shows a schematic diagram of our experimental setup. By combining two squeezed vacuum states on a 50:50 beam splitter, we generate a two-modesqueezed state. Then, delaying one of the modes using an optical delay line and then using another 50:50 beam splitter, we generate a one-dimensional cluster state - a universal resource for one-mode quantum operations. For more details regarding the principle of the timedomain-multiplexed one-dimensional cluster state, see Refs. $[4,13]$. Here we interpret the setup as sequential quantum teleportation, where the second beam splitter corresponds to a beam splitter used in the Bell measurement.

The light source of our setup is a continuous-wave Ti:sapphire laser with a wavelength $860 \mathrm{~nm}$. The $430-\mathrm{nm}$ pump beam for the optical parametric oscillator (OPO) is generated with use of a second-harmonic generator. The OPO is a triangular cavity with a periodically poled $\mathrm{KTiOPO}_{4}$ crystal inside. We select the size of the wave packet $\Delta t$ to be $40 \mathrm{~ns}$, and the corresponding optical delay line is realized by using a $12-\mathrm{m}$-free-space optical delay line. This fixes the clock frequency of our system when it is used in the quantum computation to be $25 \mathrm{MHz}$. The homodyne detectors here have a frequency bandwidth of approximately $100 \mathrm{MHz}$, which is adequate for the size of the wave packet. The initial squeezing level of each wave packet measured at the homodyne detectors is on average $-4.0 \mathrm{~dB}$ compared with the variance of the vacuum states for both $x$ quadrature and $p$ quadrature. For more details regarding the specifications of these components, see the supplemental material for Ref. [6].

The phase of the homodyne measurement is determined by the relative phase between the local oscillator and the cluster state. This phase is modified by use of an electrooptical modulator and a two-channel-signal-generator circuit. This two-channel-signal-generator circuitry is based on a field-programmable gate array (FPGA) and is used to synchronize both the changes of the measurement basis between two homodyne detectors and other control signals. The total rise time and fall time of the electrical signals sent to the electro-optical modulators are approximately 2 ns. No noticeable overshoot or ringing effects are observed in the output signals, and the jitter is observed 

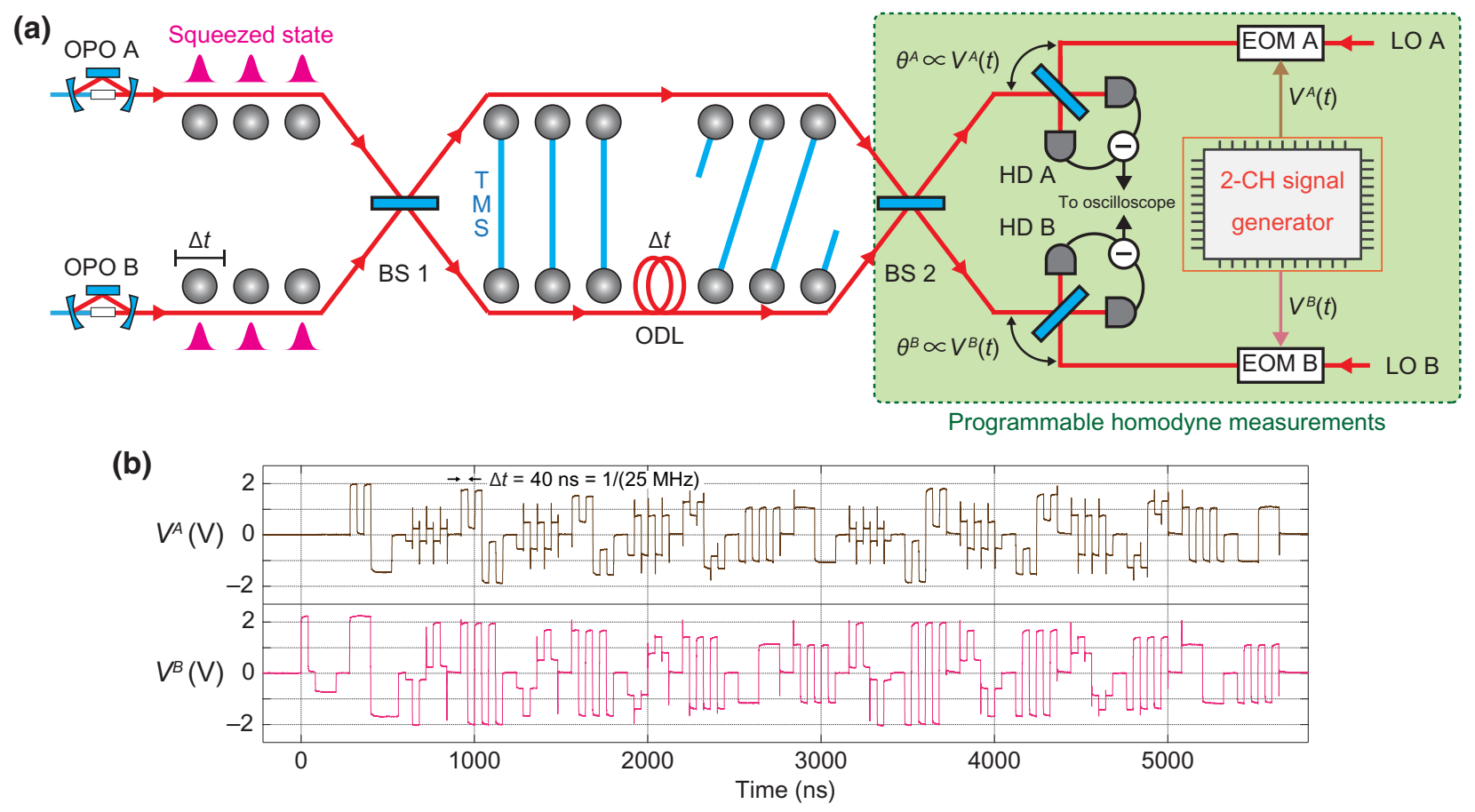

FIG. 2. The experimental setup. (a) Optical and electrical parts of the experimental setup. (b) An example of the generated signals used in the experiment. The phases of each local oscillator (LO; $\theta^{A}$ and $\theta^{B}$ ) are proportional to $V^{A}(t)$ and $V^{B}(t)$, respectively. BS, beam splitter; 2-CH, two-channel; EOM, electro-optic modulator; HD, homodyne detector; LO, local oscillator; ODL, optical delay line; OPO, optical parametric oscillator; TMS, two-mode-squeezed state; $\Delta t$, time width of the wave packet.

to be below 1 ns. Figure 2(b) shows an example of the signals generated with this circuitry used in this experiment. Implementing non-Gaussian operations additionally requires a non-Gaussian ancillary state and the implementation of adaptive homodyne measurement [22-24]. Latency plays an important role in adaptive homodyne measurements; for example, for our system, the latency must be sufficiently lower than $40 \mathrm{~ns}$. In separate work, we are developing a low-latency FPGA for such a task [16] as the latency of commercial arbitrary-waveform generators is usually too great for this application.

To stabilize the optical setup, phase reference beams whose frequencies are detuned by 126 and $256 \mathrm{kHz}$ are injected into each OPO and the interference signals are used as error signals for feedback controls. In previous generations of the time-domain-multiplexed cluster states $[5,6]$, the phase reference beams were present all the time and the measurement bases were not dynamically changed as only state verifications were done. In this experiment, however, if the phase reference beams are present, the abrupt changes of the measurement bases introduce highfrequency noise in the measurement results. To prevent that, we introduce a sample-and-hold method where the phase reference beams are turned off during the measurements [4]. The signals for controlling the sample and hold are generated from the same FPGA used in the controlling of the measurement basis, making the whole system synchronized. For every measurement, the phases of the homodyne measurements are initially locked in the $x$ basis.

The electrical signals from the homodyne detectors are recorded with an oscilloscope. We use a sampling rate of $1 \times 10^{9} \mathrm{samples} / \mathrm{s}$, and a single frame $10 \mu \mathrm{s}$ long, corresponding to 250 wave packets, is used to record experimental results of various operations. For the evaluations of each matrix element $S_{i j}$ and evaluations of each quantum correlation, 38600 events are used. After the electrical signals are recorded with the oscilloscope, they are converted to the quadrature values by numerical integration with use of a mode function. For a wave packet whose temporal index is $k$, the shape of the mode function $f_{k}(t)$ we use has the form

$f_{k}(t)= \begin{cases}(t-k \Delta t) \exp \left(-\frac{(t-k \Delta t)^{2}}{2 \tau^{2}}\right) & \text { if }|t-k \Delta t| \leq \frac{\Delta t}{2} \\ 0 & \text { otherwise, }\end{cases}$

with $\tau=5$ ns.

Feedforward operations (displacements) can be implemented by postprocessing of the homodyne-measurement data [25]. For the explicit formulae for these feedforward operations, see the supplemental material for Ref. [4]. 


\section{EVALUATION METHOD}

Figure 3 shows a schematic diagram of the evaluation method used in this experiment. As the transformations of the quadrature operators for one-mode Gaussian operations follow Eq. (1), if we ignore the displacements that are trivial, the $\mathrm{CV}$ quantum operations can be verified if we can measure the actual values of each element of the matrix $\mathbf{S}$.

If our system is a classical system, then the evaluation of the two-input-two-output linear transformation of the classical signals is relatively simple; we can simply add one signal (whose values are known) at a time and look at each output. For a quantum system, this is not the case as the quadrature operators $\hat{x}$ and $\hat{p}$ are noncommutative and the notion of knowing both the input values and the output values does not hold in quantum mechanics. Moreover, unlike the qubit system, where the measurement results are either 0 or 1 , the measurement results in the CV system take continuous values, similarly to the case of classical analog circuits.

To verify the performance of our system, we consider the two-mode-squeezed states in the system as our initial input. We use the labels "ref," "in," and "out" for the reference mode, input mode, and output mode, respectively. The input mode is the input of the quantum teleportation. When Gaussian noise is included, the general linear transformation (without displacements) in Eq. (1) has the form

$$
\left(\begin{array}{l}
\hat{x}_{\text {out }} \\
\hat{p}_{\text {out }}
\end{array}\right)=\left(\begin{array}{ll}
S_{11} & S_{12} \\
S_{21} & S_{22}
\end{array}\right)\left(\begin{array}{l}
\hat{x}_{\text {in }} \\
\hat{p}_{\text {in }}
\end{array}\right)+\hat{\mathbf{N}}
$$

where $\hat{\mathbf{N}}$ is a noise term and $\mathbf{S}$ is no longer restricted to a symplectic matrix. On the other hand, the relation between the input mode and the reference mode is given by

$$
\left(\begin{array}{c}
\hat{x}_{\text {ref }} \\
\hat{p}_{\text {ref }}
\end{array}\right)=\left(\begin{array}{c}
-\hat{x}_{\text {in }} \\
\hat{p}_{\text {in }}
\end{array}\right)+\hat{\mathbf{N}}^{\prime},
$$

where $\hat{\mathbf{N}}^{\prime}$ is a noise term due to the finite squeezing that satisfies $\left\langle\hat{\mathbf{N}}^{\prime}\right\rangle=\mathbf{0}$. Using the fact that $\left\langle\hat{x}_{\text {ref }} \hat{p}_{\text {in }}\right\rangle=\left\langle\hat{x}_{\text {in }} \hat{p}_{\text {ref }}\right\rangle=0$ for the two-mode-squeezed states, we can directly obtain the elements of $\mathbf{S}$ from the quadrature values by calculating

$$
S_{i j}=\frac{\left\langle\hat{\xi}_{\text {out }}^{(i)} \hat{\xi}_{\text {ref }}^{(j)}\right\rangle}{\left\langle\hat{\xi}_{\text {in }}^{(j)} \hat{\xi}_{\text {ref }}^{(j)}\right\rangle},
$$

where $\hat{\xi}^{(1)}=\hat{x}$ and $\hat{\xi}^{(2)}=\hat{p}$. When there are no correlations between the input mode and the reference mode, both the numerator and the denominator of Eq. (15) become 0 .

In addition to the values of the symplectic matrix $\mathbf{S}$, we also verify the amount of the quantum correlations after the quantum operations. In the experimental verifications of the cluster states [4-7,10], the van Loock-Furusawa criterion [26] is often used. This criterion is based on the measurement of the correlations between quadrature operators and their comparison with a threshold determined by the form of the correlations that are used. Although the correlations used are usually those with only $\hat{x}$ or $\hat{p}$, for our purpose, we use a more-general form [27] as the relationships between the reference mode are not limited to relationships containing only $\hat{x}$ or $\hat{p}$. For the verifications of the quantum entanglement between the reference mode and the output mode after the operations, we consider two operators, $\hat{\delta}_{1}$ and $\hat{\delta}_{2}$ :

$$
\begin{aligned}
& \hat{\delta}_{1}=\hat{\zeta}_{\text {ref }}+\hat{\zeta}_{\text {out }}, \\
& \hat{\delta}_{2}=\hat{\xi}_{\text {ref }}+\hat{\xi}_{\text {out }},
\end{aligned}
$$

where all the operators are linear in the quadrature operators. Similarly to the proof of the van Loock-Furusawa criterion, we can show that if the reference mode and the output mode are separable, then the following inequality holds:

$$
\left\langle\Delta^{2} \hat{\delta}_{1}\right\rangle+\left\langle\Delta^{2} \hat{\delta}_{2}\right\rangle \geq\left|\left\langle\left[\hat{\zeta}_{\text {ref }}, \hat{\xi}_{\text {ref }}\right]\right\rangle\right|+\left|\left\langle\left[\hat{\zeta}_{\text {in }}, \hat{\xi}_{\text {in }}\right]\right\rangle\right|
$$

where $\left\langle\Delta^{2}(\cdot)\right\rangle$ is the variance of an operator. Therefore, if we can show that the correlation between the reference mode and the output mode violates Eq. (18), then we

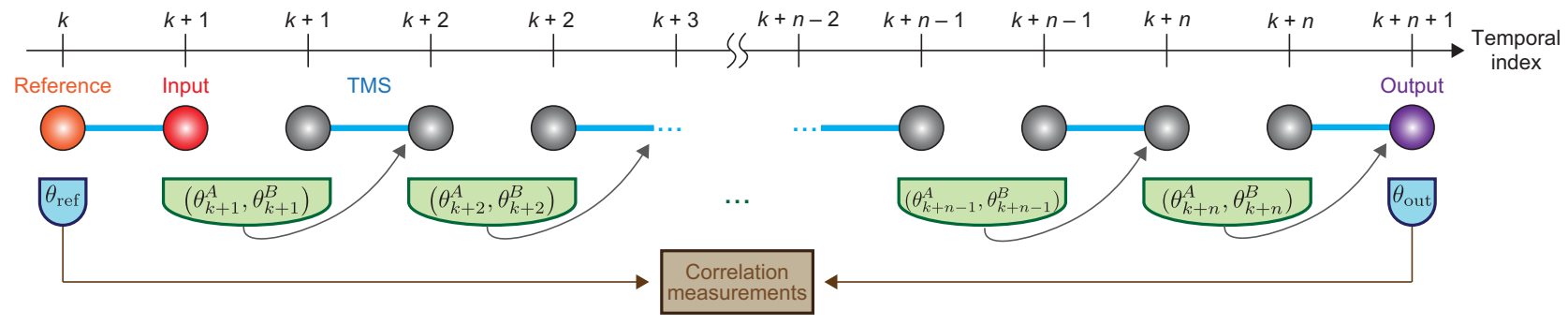

FIG. 3. Evaluation of quantum operations using quantum entanglements. Measurement bases are determined by the values of $\theta^{A}$ and $\theta^{B}$ at homodyne detectors $\mathrm{A}$ and $\mathrm{B}$. The blue detectors show the measurements of the reference mode and the output mode at bases $\theta_{\text {ref }}$ and $\theta_{\text {out }}$. TMS, two-mode-squeezed state. 
(a)

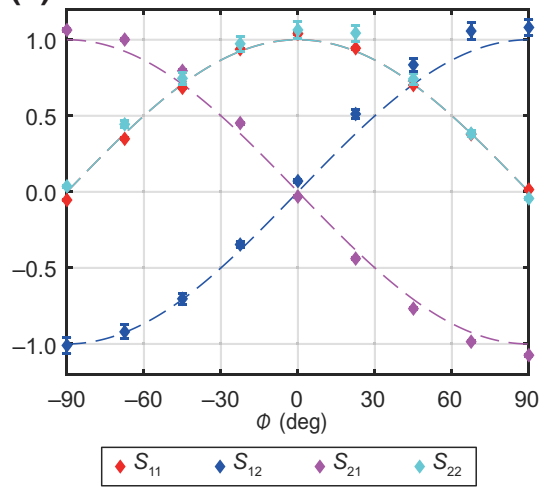

(b)

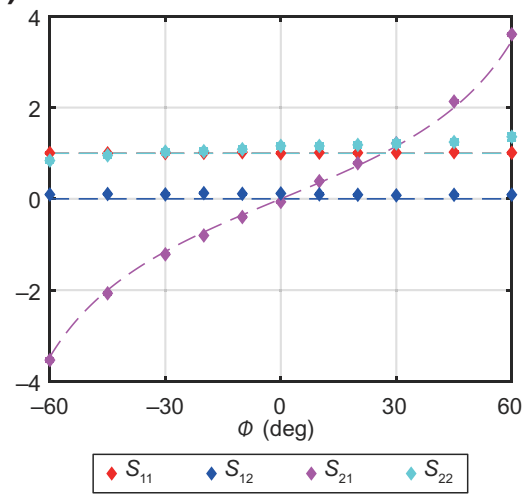

(c)

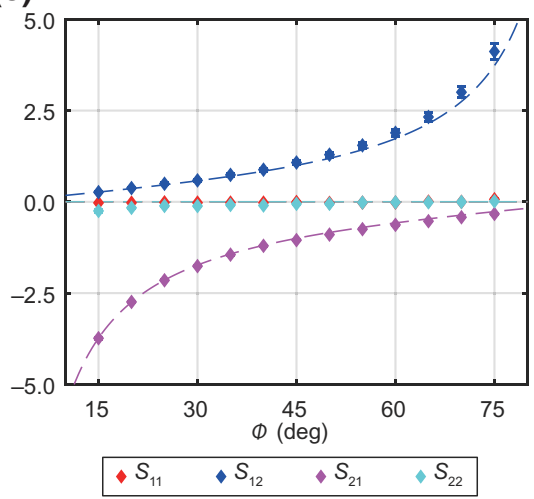

FIG. 4. Verification of single-step quantum operations for phase rotation $\mathbf{R}(\phi)$ (a), shear $\mathbf{P}(\phi)$ (b), and squeezing with $90^{\circ}$ rotation $\mathbf{R}(\pi / 2) \mathbf{S}(\phi)(\mathrm{c})$. Each point is obtained using 38600 correlation measurements. The error bars are also plotted. The dashed lines are theoretical plots.

can conclude that there is quantum entanglement between these two modes. As the van Loock-Furusawa criterion is a sufficient criterion, when Eq. (18) is not violated, the test is inconclusive.

To extract the quadrature values of the reference mode and the output mode (the blue detectors in Fig. 3), we set the measurement bases at the two homodyne detectors (HD A and HD B in Fig. 2) to the same basis. Then, from the measurement values at each homodyne detector, the quadrature values of the reference mode and the output mode are given by

$$
\begin{aligned}
& \hat{x}_{\text {ref }}(\theta)=\frac{1}{\sqrt{2}}\left[\hat{x}^{A}(\theta)-\hat{x}^{B}(\theta)\right], \\
& \hat{x}_{\text {out }}(\theta)=\frac{1}{\sqrt{2}}\left[\hat{x}^{A}(\theta)+\hat{x}^{B}(\theta)\right],
\end{aligned}
$$

which can be calculated using postprocessing.

\section{EXPERIMENTAL RESULTS}

Figure 4 shows the estimation of the symplectic matrix $\mathbf{S}$ of the various single-step one-mode Gaussian operations using the full teleportation with the measurement bases given in Eqs. (8)-(10). We observe that for these operations, the values of each component of the symplectic matrices are in good agreement with the theoretical predictions. This suggests that we succeed in the programming of the homodyne-measurement basis in the time domain. Even for the evaluation of the single-step onemode Gaussian operations, we require three measurement bases: measurement of the reference mode, measurement to implement Gaussian operations, and measurement of the output mode. Arbitrary one-mode Gaussian operations can be implemented by combinations of these Gaussian operations.
Table I shows the verifications of the quantum entanglements between the reference mode and the output mode after the quantum operations. Before the operations, the reference mode and the input mode have correlation in the form of $\hat{x}_{\text {ref }}+\hat{x}_{\text {in }}$ and $\hat{p}_{\text {ref }}-\hat{p}_{\text {in }}$. In the infinite-squeezing limit, the correlation will be perfect, and the reference mode and the input mode will be zero eigenstates of these operators - also known as nullifiers. After the quantum operations, the nullifiers are transformed and we pick $\hat{\delta}_{1}$ and $\hat{\delta}_{2}$ according to the transformed nullifiers while also minimizing the threshold. We observe that for all the operations, the variances of the nullifiers are below the vacuum variances for all three operations. Moreover, most of the operations satisfy the inseparability criterion by the van Loock-Furusawa criterion. Some of the operations that do not satisfy the van Loock-Furusawa criterion are those that involve large squeezing. In that sense, for the phase rotation $\mathbf{R}(\phi)$, the threshold level is independent of $\phi$ and the van Loock-Furusawa criterion is satisfied for all $\phi$. The values of the variances here are due to both the initial correlation between the reference mode and the input mode, and the strength of the two-mode-squeezed states used in the quantum operations, which can be improved by increasing the squeezing level and reducing the optical losses.

As a demonstration of the capability for multistep quantum operations, we implement sequential quantum teleportation (i.e., identity operation) and do an evaluation similar to that in Fig. 4 and Table I: we use one mode of the two-mode-squeezed states as inputs and implement $n$-step identity operations for various values of $n$. Then we calculate the final symplectic matrices $\mathbf{S}$ and the variances of the nullifiers. Figure 5 shows the experimental results. From the results, we observe that the values of the elements of the symplectic matrices are correct up to $n=100$. On the other hand, although the variances of the nullifiers increase above $0 \mathrm{~dB}$ in the second step due to 

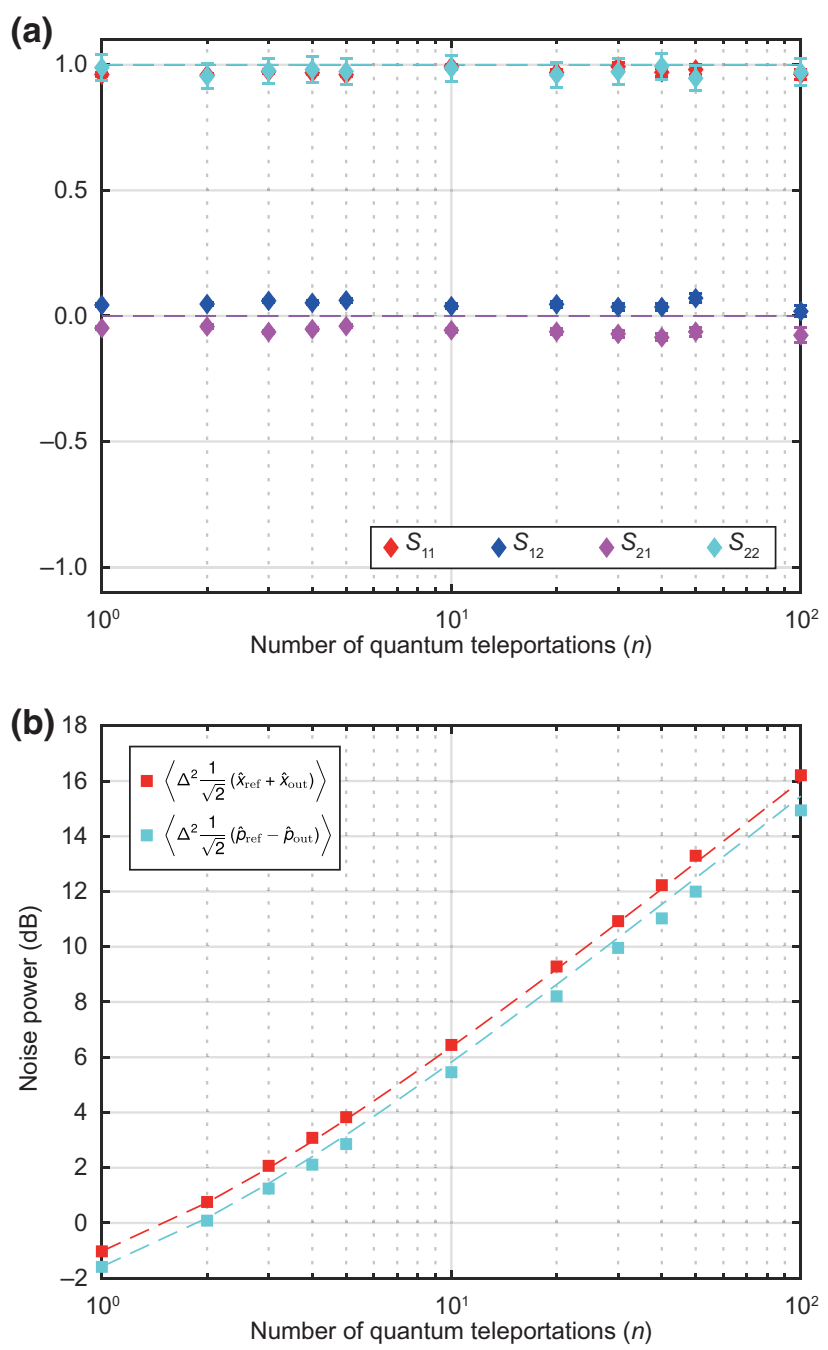

FIG. 5. Sequential quantum teleportation in the time domain. (a) Elements $S_{i j}$ of the symplectic matrix. (b) Variances of the nullifiers. The vacuum variance is $0 \mathrm{~dB}$. Each point in both figures is calculated using 38600 events. Dashed lines correspond to theoretical predictions. Predictions of the variances are based on the results of the single-step identity operation. Errors of the elements of the $\mathbf{S}$ matrix are plotted and the errors of the variances are about $\pm 0.03 \mathrm{~dB}$ for all points.

the initial finite squeezing, their dependence on $n$ is in good agreement with the theoretical predictions. The theoretical predictions are the extrapolations of the single-step teleportation $(n=1)$ with an assumption that the amount of noise added is the same for all steps. This suggests that for our current setup, the effects of the long-term stability due to the sample-and-hold method (such as those observed in Ref. [4]) are negligible up to $n=100$. Evaluation of the quantum operations by observing the added noise is an experimentally convenient approach. In followup theoretical work, we will explore the connection of this approach to a variant of the randomized-benchmarking protocol.
The main sources of the imperfection in the nullifiers in the experimental results come from the finite squeezing of the system. There are various possible sources for the observation of the finite squeezing, such as the original squeezing of the OPO, the frequency bandwidth of the OPO, the interferometric visibilities, the optical losses, the phase fluctuations, inefficiency of the photodiodes used in the homodyne detectors, and the circuit noise of the homodyne detectors. For the current setup, the effects of the interferometric visibilities and the optical losses are dominant factors for the finite squeezing. It is expected that improvements can be made with use of state-of-theart squeezed-light sources [28], where a squeezing level of around $15 \mathrm{~dB}$ has already been observed. Even then, the results here demonstrate the advantage of the timedomain multiplexing method in terms of scalability; if we were to implement a similar experiment for $n=100$ without any multiplexing, we would have to prepare hundreds of squeezed light sources, beam splitters, and homodyne detectors, stabilize the relative phases at all the beam splitters, and control the measurement bases of all the homodyne detectors with high synchronicity.

\section{DISCUSSION AND CONCLUSION}

We demonstrate one-mode Gaussian operations multiplexed in the time domain with a clock frequency of 25 $\mathrm{MHz}$. In addition to demonstrating the capability of timedomain-multiplexed cluster states to implement quantum operations in a scalable way, we also formulate a method to evaluate the performance of our system using the existing quantum entanglement. Our work is a first step toward the realization of a large-scale universal fault-tolerant quantum computer, in addition to the non-Gaussian ancillary states and nonlinear feedforward system.

The clock frequency is limited by the size of our wave packet, which in turn is limited by the frequency bandwidth of the squeezed-light sources and the homodyne detectors. Using our recently developed terahertzbandwidth squeezed-light source [29] and the technique of all-optical homodyne measurement [30,31], we expect that a clock frequency on the order of at least gigahertz can be achieved.

To achieve universal MBQC, non-Gaussian ancillary states and an adaptive homodyne-measurement system also need to be added to our setup. Both of them are being developed in separate work and are compatible with our system $[16,18]$. Therefore, the next important experimental step toward an optical quantum computer using time-domain multiplexing is the combination of non-Gaussian elements with the cluster states. There are, however, various experimental hurdles to overcome. For example, although CV cluster states are generated deterministically, optical generation of non-Gaussian states is, in general, probabilistic, meaning that an experimental 


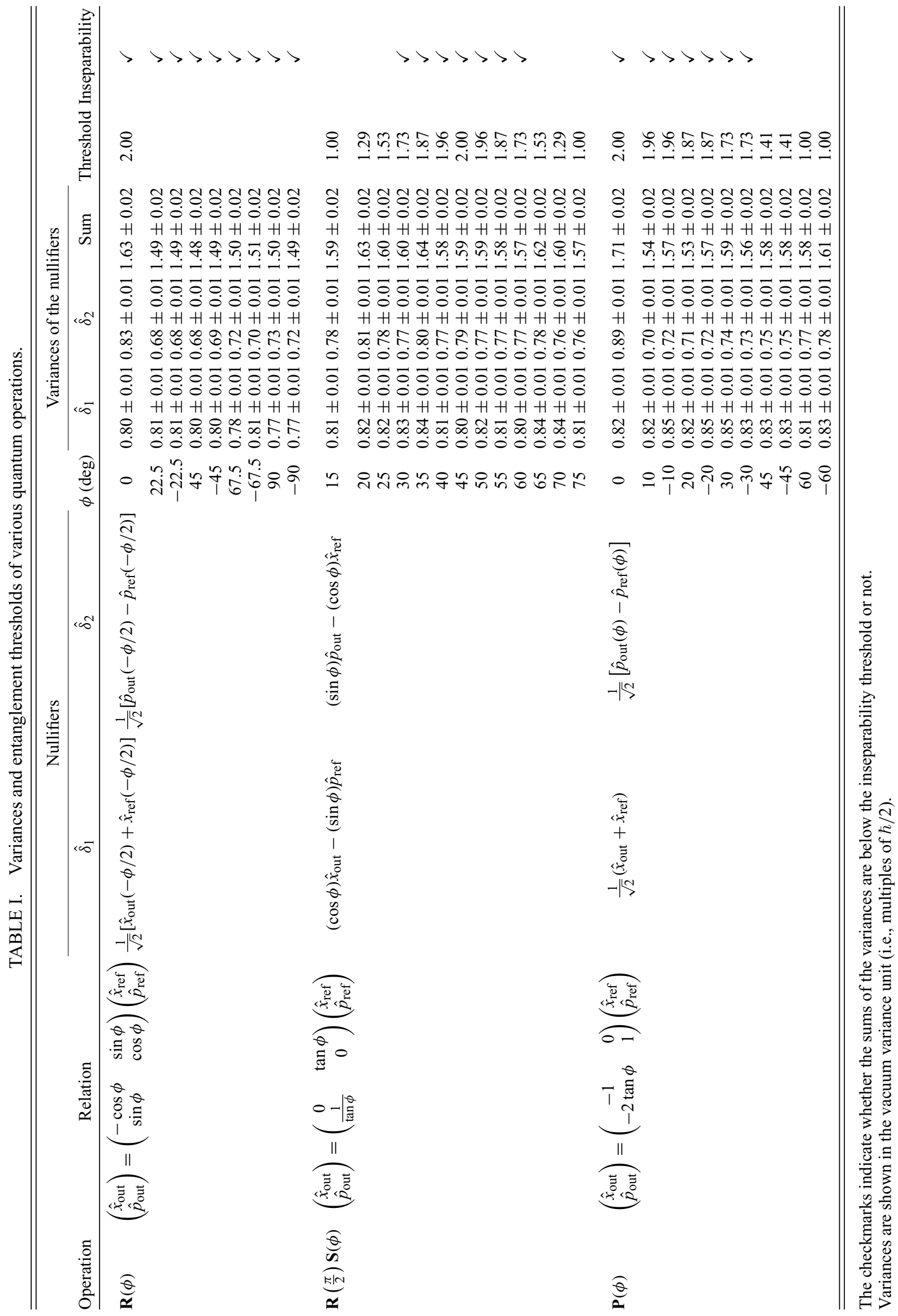


technique to join them, such as a quantum memory [32], will play an important role.

In addition to universality, fault tolerance is also required to utilize large-scale $\mathrm{CV}$ cluster states in an actual quantum computation. To achieve this, we need to increase the squeezing level of the cluster states and add the bosonic codes, of which the Gottesman-Kitaev-Preskill qubit [20] is currently the most-promising candidate. Regarding the former, current state-of-the-art squeezed-light sources [28] already admit performance close to the demands set by theoretical predictions for the fault-tolerance threshold [33-35]. For the latter, Gottesman-Kitaev-Preskill qubits have already been demonstrated in microwave [36] and ion-trapped [37] systems, and various generation methods are being explored in optical systems [38].

Finally, although still noisy, as our experiment demonstrates the platform for large-scale CV quantum computation, we expect that our work will accelerate the development of CV optical quantum computation. By extending the method in this work to two-dimensional cluster states [6,7], we can expect to see a platform for testing or benchmarking of $\mathrm{CV}$ quantum computers soon.

\section{ACKNOWLEDGMENTS}

This work was partly supported by Japan Society for the Promotion of Science KAKENHI (Grant No. 18H05207), the Core Research for Evolutional Science and Technology (CREST) (Grant No. JPMJCR15N5) and the Moonshot Research and Development Program (Grant No. JPMJMS2064) of the Japan Science and Technology Agency, UTokyo Foundation, donations from Nichia Corporation, and the Australian Research Council Centre of Excellence for Quantum Computation and Communication Technology (Project No. CE170100012). W.A. acknowledges financial support from the Japan Society for the Promotion of Science. B.C. acknowledges financial support from the Program of Excellence in Photon Science (XPS). T.N. acknowledges financial support from the Forefront Physics and Mathematics Program to Drive Transformation (FoPM). R.N.A. is supported by U.S. National Science Foundation Grant No. PHY-1630114.

W.A. and B.C. contributed equally to this work.

[1] M. A. Nielsen and I. L. Chuang, Quantum Computation and Quantum Information (Cambridge University Press, Cambridge, 2000).

[2] R. Raussendorf and H. J. Briegel, A One-Way Quantum Computer, Phys. Rev. Lett. 86, 5188 (2001).

[3] N. C. Menicucci, P. van Loock, M. Gu, C. Weedbrook, T. C. Ralph, and M. A. Nielsen, Universal Quantum Computation with Continuous-Variable Cluster States, Phys. Rev. Lett. 97, 110501 (2006).
[4] S. Yokoyama, R. Ukai, S. C. Armstrong, C. Sornphiphatphong, T. Kaji, S. Suzuki, J. Yoshikawa, H. Yonezawa, N. C. Menicucci, and A. Furusawa, Ultra-large-scale continuous-variable cluster states multiplexed in the time domain, Nat. Photonics 7, 982 (2013).

[5] J. Yoshikawa, S. Yokoyama, T. Kaji, C. Sornphiphatphong, Y. Shiozawa, K. Makino, and A. Furusawa, Invited article: Generation of one-million-mode continuous-variable cluster state by unlimited time-domain multiplexing, APL Photonics 1, 060801 (2016).

[6] W. Asavanant, Y. Shiozawa, S. Yokoyama, B. Charoensombutamon, H. Emura, R. N. Alexander, S. Takeda, J. Yoshikawa, N. C. Menicucci, H. Yonezawa, and A. Furusawa, Generation of time-domain-multiplexed twodimensional cluster state, Science 366, 373 (2019).

[7] M. V. Larsen, X. Guo, C. R. Breum, J. S. NeergaardNielsen, and U. L. Andersen, Deterministic generation of a two-dimensional cluster state, Science 366, 369 (2019).

[8] M. Gong et al., Genuine 12-Qubit Entanglement on a Superconducting Quantum Processor, Phys. Rev. Lett. 122, 110501 (2019).

[9] B. P. Lanyon, P. Jurcevic, M. Zwerger, C. Hempel, E. A. Martinez, W. Dür, H. J. Briegel, R. Blatt, and C. F. Roos, Measurement-Based Quantum Computation with Trapped Ions, Phys. Rev. Lett. 111, 210501 (2013).

[10] M. Chen, N. C. Menicucci, and O. Pfister, Experimental Realization of Multipartite Entanglement of 60 Modes of a Quantum Optical Frequency Comb, Phys. Rev. Lett. 112, 120505 (2014).

[11] P. Walther, K. J. Resch, T. Rudolph, E. Schenck, H. Weinfurter, V. Vedral, M. Aspelmeyer, and A. Zeilinger, Experimental one-way quantum computing, Nature 434, 169 (2005).

[12] R. Ukai, N. Iwata, Y. Shimokawa, S. C. Armstrong, A. Politi, J. Yoshikawa, P. van Loock, and A. Furusawa, Demonstration of Unconditional One-Way Quantum Computations for Continuous Variables, Phys. Rev. Lett. 106, 240504 (2011).

[13] N. C. Menicucci, Temporal-mode continuous-variable cluster states using linear optics, Phys. Rev. A 83, 062314 (2011).

[14] E. Knill, D. Leibfried, R. Reichle, J. Britton, R. B. Blakestad, J. D. Jost, C. Langer, R. Ozeri, S. Seidelin, and D. J. Wineland, Randomized benchmarking of quantum gates, Phys. Rev. A 77, 012307 (2008).

[15] R. N. Alexander, P. S. Turner, and S. D. Bartlett, Randomized benchmarking in measurement-based quantum computing, Phys. Rev. A 94, 032303 (2016).

[16] A. Sakaguchi, H. Ogawa, M. Kobayashi, S. Suzuki, H. Yonezawa, E. Huntington, S. Takeda, J. Yoshikawa, and A. Furusawa, in Conference on Lasers and Electro-Optics (Optical Society of America, 2018), p. FTu4G.3.

[17] S. Konno, A. Sakaguchi, W. Asavanant, H. Ogawa, M. Kobayashi, P. Marek, R. Filip, J. Yoshikawa, and A. Furusawa, Nonlinear Squeezing for Measurement-Based NonGaussian Operations in Time Domain, Phys. Rev. Appl. 15, 024024 (2021).

[18] M. Yukawa, K. Miyata, T. Mizuta, H. Yonezawa, P. Marek, R. Filip, and A. Furusawa, Generating superposition of up-to three photons for continuous variable quantum information processing, Opt. Express 21, 5529 (2013). 
[19] S. Lloyd and S. L. Braunstein, Quantum Computation Over Continuous Variables, Phys. Rev. Lett. 82, 1784 (1999).

[20] D. Gottesman, A. Kitaev, and J. Preskill, Encoding a qubit in an oscillator, Phys. Rev. A 64, 012310 (2001).

[21] B. Q. Baragiola, G. Pantaleoni, R. N. Alexander, A. Karanjai, and N. C. Menicucci, All-Gaussian Universality and Fault Tolerance with the Gottesman-Kitaev-Preskill Code, Phys. Rev. Lett. 123, 200502 (2019).

[22] P. Marek, R. Filip, H. Ogawa, A. Sakaguchi, S. Takeda, J. Yoshikawa, and A. Furusawa, General implementation of arbitrary nonlinear quadrature phase gates, Phys. Rev. A 97, 022329 (2018).

[23] K. Miyata, H. Ogawa, P. Marek, R. Filip, H. Yonezawa, J. Yoshikawa, and A. Furusawa, Implementation of a quantum cubic gate by an adaptive non-gaussian measurement, Phys. Rev. A 93, 022301 (2016).

[24] S. Konno, W. Asavanant, K. Fukui, A. Sakaguchi, F. Hanamura, P. Marek, R. Filip, J. Yoshikawa, and A. Furusawa, Non-Clifford gate on optical qubits by nonlinear feedforward, arXiv:2103.10644 (2021).

[25] R. N. Alexander, S. Yokoyama, A. Furusawa, and N. C. Menicucci, Universal quantum computation with temporalmode bilayer square lattices, Phys. Rev. A 97, 032302 (2018).

[26] P. van Loock and A. Furusawa, Detecting genuine multipartite continuous-variable entanglement, Phys. Rev. A 67, 052315 (2003).

[27] R. Ukai, Multi-Step Multi-Input One-Way Quantum Information Processing with Spatial and Temporal Modes of Light (Springer Japan, Tokyo, 2015).

[28] H. Vahlbruch, M. Mehmet, K. Danzmann, and R. Schnabel, Detection of $15 \mathrm{~dB}$ Squeezed States of Light and their Application for the Absolute Calibration of Photoelectric Quantum Efficiency, Phys. Rev. Lett. 117, 110801 (2016).

[29] T. Kashiwazaki, N. Takanashi, T. Yamashima, T. Kazama, K. Enbutsu, R. Kasahara, T. Umeki, and A. Furusawa, Continuous-wave 6-dB-squeezed light with $2.5-\mathrm{THz}-$ bandwidth from single-mode PPLN waveguide, APL Photonics 5, 036104 (2020).

[30] Y. Shaked, Y. Michael, R. Z. Vered, L. Bello, M. Rosenbluh, and A. Pe'er, Lifting the bandwidth limit of optical homodyne measurement with broadband parametric amplification, Nat. Commun. 9, 609 (2018).

[31] N. Takanashi, A. Inoue, T. Kashiwazaki, T. Kazama, K. Enbutsu, R. Kasahara, T. Umeki, and A. Furusawa, Alloptical phase-sensitive detection for ultra-fast quantum computation, Opt. Express 28, 34916 (2020).

[32] F. Okamoto, M. Endo, M. Matsuyama, Y. Ishizuka, Y. Liu, R. Sakakibara, Y. Hashimoto, J. Yoshikawa, P. van Loock, and A. Furusawa, Phase Locking between Two All-Optical Quantum Memories, Phys. Rev. Lett. 125, 260508 (2020).

[33] N. C. Menicucci, Fault-Tolerant Measurement-Based Quantum Computing with Continuous-Variable Cluster States, Phys. Rev. Lett. 112, 120504 (2014).

[34] K. Fukui, A. Tomita, A. Okamoto, and K. Fujii, HighThreshold Fault-Tolerant Quantum Computation with Analog Quantum Error Correction, Phys. Rev. X 8, 021054 (2018).

[35] B. W. Walshe, L. J. Mensen, B. Q. Baragiola, and N. C. Menicucci, Robust fault tolerance for continuous-variable cluster states with excess antisqueezing, Phys. Rev. A 100, 010301 (2019).

[36] P. Campagne-Ibarcq, A. Eickbusch, S. Touzard, E. ZalysGeller, N. E. Frattini, V. V. Sivak, P. Reinhold, S. Puri, S. Shankar, R. J. Schoelkopf, L. Frunzio, M. Mirrahimi, and M. H. Devoret, Quantum error correction of a qubit encoded in grid states of an oscillator, Nature 584, 368 (2020).

[37] C. Flühmann, T. L. Nguyen, M. Marinelli, V. Negnevitsky, K. Mehta, and J. P. Home, Encoding a qubit in a trapped-ion mechanical oscillator, Nature 566, 513 (2019).

[38] I. Tzitrin, J. E. Bourassa, N. C. Menicucci, and K. K. Sabapathy, Progress towards practical qubit computation using approximate Gottesman-Kitaev-Preskill codes, Phys. Rev. A 101, 032315 (2020). 